\title{
Entanglement Reciprocation between Qubits and Continuous Variables
}

\author{
Jinhyoung Lee, ${ }^{1}$ M. Paternostro, ${ }^{2}$ M. S. Kim, ${ }^{2}$ and S. Bose ${ }^{3}$ \\ ${ }^{1}$ Department of Physics and Quantum Photonic Science Research Center, Hanyang University, Seoul 133-791, Korea \\ ${ }^{2}$ School of Mathematics and Physics, Queen's University, Belfast BT7 1NN, United Kingdom \\ ${ }^{3}$ Department of Physics and Astronomy, University College London, London WC1E 6BT, United Kingdom
}

(Received 20 September 2005; published 28 February 2006)

\begin{abstract}
We investigate how entanglement can be transferred between qubits and continuous-variable (CV) systems. We find that one ebit borne in maximally entangled qubits can be fully transferred to two CV systems which are initially prepared in a pure separable Gaussian field with high excitation. We show that it is possible to retrieve the entanglement back to qubits from the entangled CV systems. The deposition of multiple ebits from qubits to the initially separable CV systems is also pointed out. We show that the entanglement transfer and retrieval are done at a quasisteady state.
\end{abstract}

DOI: 10.1103/PhysRevLett.96.080501

PACS numbers: 03.67.Mn, 03.67.Hk, 42.50.Ct

Quantum information processing (QIP) has been extensively studied for a qubit system which is a quantum extension of a bit, spanning two-dimensional Hilbert space. A qubit is realized by a spin, a two-level atom, the polarization of a photon, and a superconductor among others. A two-dimensional system is mathematically handy and logically easy to treat. On the other hand, many continuous-variable (CV) physical systems such as a harmonic oscillator and a light field, which are defined in infinite-dimensional Hilbert space, have also attracted considerable attention for other practical reasons. While qubit and CV systems are nearly always treated separately, there is a good reason to believe that a study of their interface may result in synergy for the implementation of QIP. There have been some pilot works on how to entangle two separate qubits by an entangled Gaussian field [1-3]. In this Letter, we ask the interesting questions of how easy it is to deposit the entanglement of two qubits to two coherent states and retrieve quantum entanglement back to the qubits.

When two maximally entangled two-level atoms are sent to two respective cavities initially prepared in vacuum, after the Rabi time the maximal entanglement is fully transferred to the cavity fields $[4,5]$. Here the interaction is assumed resonant and the cavities are lossless. Essentially, in the above transfer the cavity does not behave as a true CV system, as only cavity states $|0\rangle$ and $|1\rangle$ play a part. Adding an extra excitation in a cavity initially in vacuum will be noticeable. However, if the cavities are prepared with coherent fields of large amplitudes, will the atom's depositing extra excitation still be visible to show the entanglement of ebit? Can the entanglement be retrieved by the next set of atoms? Answering the questions, we find that it is possible to perfectly deposit entanglement to initial coherent fields and retrieve them back at a quasisteady state. We also show the possibility to extend the memory to multidimensional states. The cavities thus act as a memory for entanglement, differently from the usual perspective where the atoms are designed to memorize the quantum state. Our approach is based on the use of the resonant Jaynes-Cummings (JC) model [6]. This is not a limitation, as this model has been proven to be naturally valid in many physical situations in which coherent exchange of excitations between spinlike particles and bosons are involved [7].

Model. - Let us consider two atoms in the triplet state

$$
|\psi(0)\rangle_{a}=\frac{1}{\sqrt{2}}\left(|e\rangle_{1}|g\rangle_{2}+|g\rangle_{1}|e\rangle_{2}\right)
$$

where $|e\rangle$ and $|g\rangle$ stand for the excited and ground states of the atom. This state is maximally entangled and is said to carry one ebit of entanglement. The two atoms enter their respective cavities which are initially prepared with coherent states. For convenience, we assume that the amplitudes of the coherent states are $\alpha \in \mathbb{R}$ throughout the Letter. The initial state for the atoms and fields is $|\Psi(0)\rangle_{a f}=|\psi(0)\rangle_{a}|\alpha\rangle_{1}|\alpha\rangle_{2}$.

We consider how much of the atomic entanglement, in units of ebit, is transferred to the infinite-dimensional cavity fields by their resonant interaction. Under the rotating wave approximation, the interaction Hamiltonian $\hat{H}=$ $\hbar \lambda\left(\hat{a}^{\dagger}|g\rangle\langle e|+\hat{a}| e\rangle\langle g|\right)$, where the bosonic creation and annihilation operators are denoted by $\hat{a}^{\dagger}$ and $\hat{a}$, respectively, and the coupling between the field and the atom by $\lambda$. The evolution of the atom and field state is then determined by the propagation operator $\hat{U}=\hat{U}_{1} \otimes \hat{U}_{2}$, where, in atomic basis $\langle e|=(1,0)$ and $\langle g|=(0,1), \quad \hat{U}_{i}=$ $\left\{\left\{\hat{U}_{11}^{(i)}, \hat{U}_{12}^{(i)}\right\},\left\{\hat{U}_{21}^{(i)}, \hat{U}_{22}^{(i)}\right\}\right\}$. The operators at the interaction time $t$ read [8]

$$
\begin{gathered}
\hat{U}_{11}^{(i)}=\cos \lambda t \sqrt{\hat{a}_{i} \hat{a}_{i}^{\dagger}}, \quad \hat{U}_{12}^{(i)}=-i \hat{a}_{i} \frac{\sin \lambda t \sqrt{\hat{a}_{i}^{\dagger} \hat{a}_{i}}}{\sqrt{\hat{a}_{i}^{\dagger} \hat{a}_{i}}}, \\
\hat{U}_{21}^{(i)}=-i \hat{a}_{i}^{\dagger} \frac{\sin \lambda t \sqrt{\hat{a}_{i} \hat{a}_{i}^{\dagger}}}{\sqrt{\hat{a}_{i} \hat{a}_{i}^{\dagger}}}, \quad \hat{U}_{22}^{(i)}=\cos \lambda t \sqrt{\hat{a}_{i}^{\dagger} \hat{a}_{i}} .
\end{gathered}
$$

After the interaction, the atom-field state evolves to $\hat{U}|\Psi(0)\rangle_{a f}$. Here, we postselect the cavity field conditioned 
on two atoms leaving the cavities in their ground states. The main reason of the postselection is to bring the cavity field to a pure state, whose measure of entanglement is the von Neumann entropy of the reduced density operator. While in this Letter we are interested in a possibility for qubits to deposit one complete ebit to a large CV system, there is no measure or criterion of entanglement for a general CV state. The field state after postselection is

$$
|\psi(1)\rangle_{f}=\frac{\mathcal{N}}{\sqrt{2}}\left(\hat{U}_{21}^{(1)} \hat{U}_{22}^{(2)}+\hat{U}_{22}^{(1)} \hat{U}_{21}^{(2)}\right)|\alpha\rangle_{1}|\alpha\rangle_{2}
$$

The normalization constant is denoted by $\mathcal{N}$, and the coherent state is expanded [9] such as $|\alpha\rangle=\sum_{m} C_{m}|m\rangle$, where $C_{m}=\alpha^{m} e^{-\alpha^{2} / 2} / \sqrt{m}$ ! gives a Poissonian weight with the average photon number $\bar{n}=\alpha^{2}$. Substituting these into Eq. (3), we find $|\psi(1)\rangle_{f}=\sum_{n, m=0}^{\infty} C_{n, m}|n\rangle_{1}|m\rangle_{2}$ with $C_{n, m}=\frac{-i \mathcal{N} e^{-\alpha^{2}}}{\alpha \sqrt{2}}\left[\frac{\alpha^{n+m} \sin (\lambda t \sqrt{n}) \cos (\lambda t \sqrt{m})}{\sqrt{m !(n-1) !}}+n \leftrightarrow m\right]$.

Entanglement transfer from qubits to $C V$ system.-The atoms initially have one ebit as they are maximally entangled. We would like to know how much ebit is transferred to the cavity fields by the resonant interaction. As the cavity fields are in a pure state $|\psi(1)\rangle_{f}$, the amount of ebit $\mathcal{E}$ is calculated by $\mathcal{E}=-\operatorname{Tr} \hat{\rho}_{f 1} \log _{2} \hat{\rho}_{f 1}$ where the reduced density operator for the cavity field 1 is

$$
\hat{\rho}_{1}=\operatorname{Tr}_{2} \hat{\rho}_{f}=\sum_{m, n, n^{\prime}} C_{n, m} C_{n^{\prime}, m}^{*}|n\rangle\left\langle n^{\prime}\right| .
$$

In Fig. 1(a) we plot $\mathcal{E}$ against $\alpha$ and the interaction time $\lambda t$ (unit of $\pi$ ). For $\alpha=0$, we know $\mathcal{E}$ can be 1 for sure. Figure 1(b) shows the probability of the atoms leaving the cavities in the ground states. When $\alpha<1$, an oscillating behavior is observed in the degree of entanglement as well as in the atomic population. On the other hand, it is interesting to note that when $\alpha$ is large the cavities are with complete ebit whenever the atoms leave the cavities in their ground states except the first moments of oscillations. We can analyze this by showing that $\left|\phi_{1}\right\rangle \equiv \hat{U}_{21}|\alpha\rangle$ is orthogonal to $\left|\phi_{2}\right\rangle \equiv \hat{U}_{22}|\alpha\rangle$ in Eq. (3), i.e., $v_{0} \equiv$ $\left|\left\langle\phi_{1} \mid \phi_{2}\right\rangle\right|=\frac{e^{-\alpha^{2}}}{2} \sum_{n} \frac{\sqrt{n}}{\alpha} \frac{\alpha^{2 n}}{n !} \sin 2 \lambda t \sqrt{n}=0$. If so, state (3) becomes a maximally entangled qubit state as it will be an equally weighted superposition of two orthogonal com-
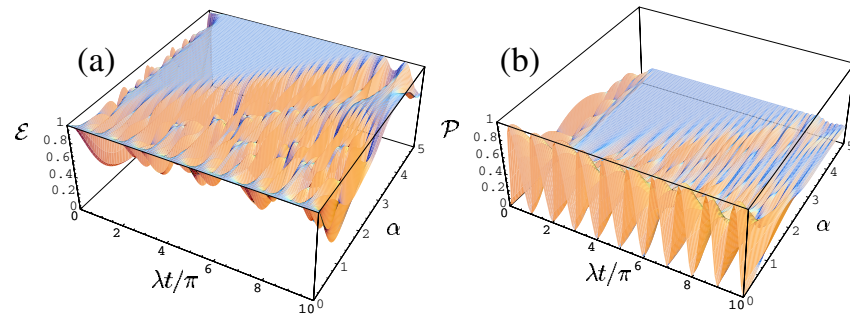

FIG. 1 (color online). (a) Degree of entanglement for the cavity field depending on the interaction time $\lambda t$ (in units of $\pi$ ) and the amplitude $\alpha$ of the initial coherent state. (b) Probability of finding the atoms leaving the cavities in their ground states. posite states. In the limit of $\alpha^{2} \gg 1$, the Poissonian distribution is replaced by a Gaussian distribution over the variable $n$ with mean value and variance equal to $\alpha^{2}$ [9] so that $C_{n}^{2} \equiv e^{-\alpha^{2}} \frac{\alpha^{2 n}}{n !} \approx \frac{1}{\sqrt{2 \pi \alpha^{2}}} e^{-\left(n-\alpha^{2}\right)^{2} / 2 \alpha^{2}}$. Taking into account the largely contributing terms of $\sqrt{n}$, i.e., those of $n$ near the peak $\alpha^{2}$, we have $\sqrt{n}=\sqrt{\alpha^{2}+\left(n-\alpha^{2}\right)} \approx$ $\alpha\left(1+\frac{n-\alpha^{2}}{2 \alpha^{2}}\right)$. Finally, the summation over $n$ is replaced by an integration in terms of $x=\left(n-\alpha^{2}\right) / \alpha$ and the integration region is extended to $(-\infty, \infty)$. We then immediately recognize $v_{0}$ as a Fourier transformation of a Gaussian function:

$$
v_{0} \propto\left(\sin 2 \alpha \lambda t+\frac{\lambda t}{2 \alpha} \cos 2 \lambda \alpha t\right) e^{-\lambda^{2} t^{2} / 2},
$$

which decreases exponentially to zero and the two states become orthogonal to each other exponentially with regard to the interaction time. This shows the transfer of a complete ebit from two qubits to a CV system of a large amplitude. It is easy to show that the postselection probability is $25 \%$ for the limit considered here.

Using the same analogy to prove their orthogonality, we can show that $\left\langle\phi_{1} \mid \phi_{1}\right\rangle=\left\langle\phi_{2} \mid \phi_{2}\right\rangle$ for the same limit. Suppose the initial atomic state was prepared not in the perfect triplet state (1) but in a partially entangled mixed or pure state. If we again assume the case of postselecting atoms in their ground states, from the earlier analysis we know that the atom initially in $|e\rangle$ will take the initial coherent field to $\left|\phi_{1}\right\rangle$ and $|g\rangle$ to $\left|\phi_{2}\right\rangle$. As the two field state bases are orthogonal with the same weight, it is straightforward to show that the field state collapses to the state which bears the same amount of entanglement as in the initial atomic qubits. This shows the perfect transfer of initial entanglement to a CV system [10].

In order to see the transfer of the ebit, we took a limit to ignore the discrete nature of photons. However, it is interesting to note that we need to recover the discrete nature to explain the revival of the oscillatory behavior in entanglement as shown in Fig. 1(a). The revival occurs when the sinusoidal functions $v_{0}$ are in phase. The significant contributions of the sinusoidal functions come from around the peak of the Poissonian distribution. At the peak of the revival time, $t_{r}: 2 \lambda t_{r}\left(\sqrt{\alpha^{2}}-\sqrt{\alpha^{2}-1}\right)=2 \pi$. Taking only the first two terms of the binomial expansion of the square root, we find the revival time $t_{r}=2 \alpha \pi / \lambda$. In fact, the dynamics of entanglement follows the well-known collapse-revival dynamics of JC model.

Entanglement retrievals. - We have seen that the qubits can transfer a complete ebit to a CV system conditionally. The next question is this: Will it be possible for the qubits to retrieve the ebit from the CV system? In order to solve this problem, we take the second set of atoms initially prepared in their ground states to send through the respective cavities which are in $|\psi(1)\rangle_{f}$. According to the earlier discussions, after the interaction time of $t^{\prime}$, the atom-field state becomes 


$$
|\Psi(2)\rangle_{a-f}=\left(\begin{array}{cc}
\hat{U}_{12}^{(1)} & \hat{U}_{12}^{(2)} \\
\hat{U}_{12}^{(1)} & \hat{U}_{22}^{(2)} \\
\hat{U}_{22}^{(1)} & \hat{U}_{12}^{(2)} \\
\hat{U}_{22}^{(1)} & \hat{U}_{22}^{(2)}
\end{array}\right)|\psi(1)\rangle_{f}=\sum_{n, m=0}^{\infty} \mathbf{V}_{a}|n\rangle_{1}|m\rangle_{2},
$$

with the matrix

$$
\mathbf{V}_{a}=\left(\begin{array}{c}
-\sin \left(\lambda t^{\prime} \sqrt{n+1}\right) \sin \left(\lambda t^{\prime} \sqrt{m+1}\right) C_{n+1, m+1} \\
-i \sin \left(\lambda t^{\prime} \sqrt{n+1}\right) \cos \left(\lambda t^{\prime} \sqrt{m}\right) C_{n+1, m} \\
-i \cos \left(\lambda t^{\prime} \sqrt{n}\right) \sin \left(\lambda t^{\prime} \sqrt{m+1}\right) C_{n, m+1} \\
\cos \left(\lambda t^{\prime} \sqrt{n}\right) \cos \left(\lambda t^{\prime} \sqrt{m}\right) C_{n, m}
\end{array}\right)
$$

In order to investigate how much of the field entanglement deposited by the first set of atoms would be transferred to the second set, we trace $|\Psi(2)\rangle_{a f}$ over the field variables and find the state of the atoms: $\hat{\rho}_{a}=\sum_{n, m} \mathbf{V}_{a} \mathbf{V}_{a}^{\dagger}$. The degree of entanglement for the two atoms is found using the log negativity [11] of the partial transposition of the density operator $\hat{\rho}_{a}$ and plotted in Fig. 2(a) as a function of the interaction times $\lambda t^{\prime}$. It is seen that, for nonzero $\alpha$, the $\mathrm{CV}$ fields are not able to transfer the complete ebit to the atoms. However, we cannot simply say that it is possible to transfer an ebit from a qubit system to a CV system while the converse is not true. The reason is that the qubit $\rightarrow \mathrm{CV}$ transfer of an ebit was conditioned on the qubits in their ground states, having lost their entanglement.

In order to improve the degree of entanglement transferred to the atoms, we consider an orthogonal measurement of $\left\{\hat{P}_{\alpha}^{(i)}, \hat{Q}_{\alpha}^{(i)}=1-\hat{P}_{\alpha}^{(i)}\right\}$, where $\hat{P}_{\alpha}^{(i)}$ is the projection onto the coherent state of its amplitude $\alpha$. In Fig. 2(b), the degree of entanglement for the atoms is plotted, conditioned on the fields in $\hat{P}_{\alpha}^{(1)} \hat{P}_{\alpha}^{(2)}$ for interaction times $t^{\prime}=t$. It is interesting to see the complete entanglement transfer for a CV system to a qubit system. We analyze this as follows. By postselecting the event of $(\alpha, \alpha)$ after the interaction time $t^{\prime}=t$, the atomic state becomes
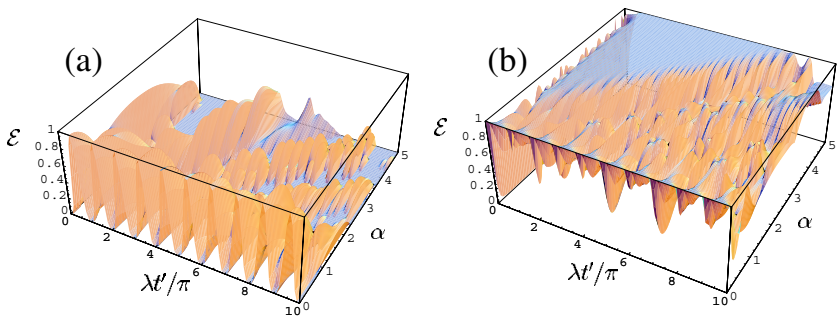

FIG. 2 (color online). (a) Degree of entanglement for the second pair of atoms depending on $\alpha$ and the interaction time $\lambda t^{\prime}=\lambda t$. (b) Analogous plot for the postselected event $(\alpha, \alpha)$. Note that the shape resembles Fig. 1(a), the degree of entanglement for the cavity field.

$$
|\psi(2)\rangle_{a}=\frac{\mathcal{N}^{\prime}}{\sqrt{2}}\left(\begin{array}{c}
2 v_{1} v_{2} \\
v_{1} v_{4}+v_{2} v_{3} \\
v_{3} v_{2}+v_{4} v_{1} \\
2 v_{3} v_{4}
\end{array}\right),
$$

where $v_{1}=\left\langle\alpha\left|\hat{U}_{12} \hat{U}_{21}\right| \alpha\right\rangle, \quad v_{2}=\left\langle\alpha\left|\hat{U}_{12} \hat{U}_{22}\right| \alpha\right\rangle, \quad v_{3}=$ $\left\langle\alpha\left|\hat{U}_{22} \hat{U}_{21}\right| \alpha\right\rangle$, and $v_{4}=\left\langle\alpha\left|\hat{U}_{22} \hat{U}_{22}\right| \alpha\right\rangle . \quad \mathcal{N}^{\prime}$ is the new normalization factor. Using the same approximation leading to Eq. (5), we find that $v_{1,4} \approx \frac{1}{2} \times$ $\left[ \pm 1+\cos (2 \alpha \lambda t) e^{-(\lambda t)^{2} / 2}\right]$, where the $-\operatorname{sign}$ is for $v_{1}$ and the + sign is for $v_{4}$, and $v_{2}=v_{3} \approx \mathcal{O}(t) e^{-(\lambda t)^{2} / 2}$, where $\mathcal{O}(t)$ is a linear sum of sinusoidal functions. In the long time limit $\lambda t \gg 1, v_{2}=v_{3} \rightarrow 0$ while $v_{1}=$ $-v_{4}=-\frac{1}{2}$. We can see that state (8) is now a maximally entangled triplet state (1), which has been perfectly retrieved after the interactions with the cavity fields. This can be inferred from the analysis of the entanglement between the second pair of atoms shown in Fig. 2(b). Surprisingly, the probability of getting the coherent state is as high as $50 \%$, in this limit.

We sketch a scheme to project the field state to $\hat{P}_{\alpha}=$ $|\alpha\rangle\langle\alpha|$ as follows. Observing the identity $|\alpha\rangle\langle\alpha|=$ $\hat{D}(\alpha)|0\rangle\langle 0| \hat{D}^{\dagger}(\alpha)$ with $\hat{D}(\alpha)$ the displacement operator [9], we suggest to project the field to the vacuum after displacing it by the amplitude $-\alpha$. After the interaction with the qubit pair, a coherent field of amplitude can be injected into the cavities, thus implementing an effective displacement operation [12]. We then electrically release one of the mirrors of a cavity (by effectively lowering its quality factor) so to allow the field to leak out. By considering the events in which no photon is detected out of the cavities, the effective projection is implemented, which realizes the measurement $\otimes_{j=1}^{2} \hat{P}_{\alpha}^{(j)}$. However, a no-click event could result from a nonunit quantum efficiency detector missing photons coming out of the cavity. Assuming an imperfect but highly efficient detector, we have calculated the effect of missing one photon by one of the detectors and found that the entanglement of the pair of atoms, in this circumstance, exhibits a short plateau corresponding to an ebit being shared between the qubits. This occurs in coincidence of the collapse part in Fig. 2(b). Thus, by properly choosing the interaction times, a reliable entanglement retrieval can still be obtained.

It is worth stressing that the results presented in this work are a feature of the coherent state used as a memory for entanglement. Indeed, we have checked that, by considering initial thermal states for the fields and applying the protocol in the previous paragraphs, the initial ebit in the qubit state can never be completely deposited in the CV state and, consequently, retrieved from it. Obviously, in order to investigate the entanglement deposit, one faces the hard problem of quantifying the entanglement in a two-mode non-Gaussian state. This difficulty has been bypassed adopting the technique described in Ref. [14] based on the projection onto a subspace spanned by the 
bidimensional bases $\{|n\rangle,|n+1\rangle\}_{j}$ with $j=1,2$. The entanglement within the resulting projected state is then averaged over thermal weighting functions characterized by their mean photon number $\bar{n}$. This provides us with a lower bound to the entanglement in the two-mode nonGaussian state. It is thus straightforward to see that the perfect deposit-retrieval process is possible just for the trivial case of $\bar{n}=0$ for the initial fields.

An interesting question to ask now is what happens when a series of atom pairs, each in the state $|\psi(0)\rangle_{a}$, interact with the cavity fields (in the usual setting of one atom with each cavity field). We found that, for example, for $\alpha=4.5$ and if the first pair of atoms had interacted for a time $t_{1}=6.47 / \lambda$ (which deposits an ebit of entanglement), then a second pair of atoms interacting for a time $t_{2}=11.04 / \lambda$ deposits another ebit, and a third pair interacting for a time $t_{3}=3.24 / \lambda$ deposits yet another ebit. Each of these events has a success probability of $\sim 25 \%$ and are robust to small variations in $t_{i}$, as before. This contrasts the case of the cavities starting in $|00\rangle_{12}$ where incommensurate Rabi frequencies prevent the deposition of more than one ebit. The cavities in our case can thus serve as "stationary" reservoirs for multiple ebits supplied by atom pairs in the form of "flying" qubits, which may be difficult to hold in other situations. In addition, these multiple ebit entangled cavity states may be directly used for teleportation of higher dimensional states. Using $\hat{P}_{\alpha}$, $\hat{Q}_{\alpha}$ allows the retrieval of 1.82 and 1.91 ebits at optimized times from the 2- and 3-ebit entangled cavity states, respectively.

We would like to point out that our approach is quite setup independent. The very recent progress in micro- and nanofabrication of integrated cavity-qubit systems in the semiconductor and superconducting domain [15] and the readily available sources of coherent states in many ranges of frequency make our proposal adaptable to different physical situations. However, considerations related to the quality of the cavities will decide the most suitable setup. Obviously, an implementation based on microwave cavity-quantum electrodynamics (QED) would be the most natural choice [13]. In this case, indeed, a long photon lifetime within the cavity $(\sim 1 \mathrm{~ms})$ allows for coherent atom-cavity dynamics lasting many Rabi floppings [13]. For $\alpha \geq 2$, about 2 Rabi floppings (each lasting $\sim 10^{-2} \mathrm{~ms}$ ) are sufficient to deposit (retrieve) a full ebit, thus making our protocol robust against cavity losses in a microwave cavity-QED or a circuit-QED scenario (see Wallraff et al. in [15]). On the other hand, an important time scale is given by the waiting time between the deposit and retrieval processes. During this period, photon losses could spoil the $\mathrm{CV}$ entangled state. The experimental value for the probability $p$ of losing a photon is $\approx 10 \%$ during a waiting time of about 10 Rabi floppings. In this case (considering a single photon loss from one of the cavities and neglecting further losses), we have checked the entan- glement of the retrieving pair of atoms in a mixed state and found its log negativity as large as $\sim 0.9$.

Remarks. -We have considered interfaces between two heterodimensional systems. An ebit can be transferred to a $\mathrm{CV}$ system from a qubit system and back in a conditional way. An interesting point is that the transfer happens in the quasisteady state. We also found an interesting analogy between the entanglement reciprocation and the collapse and revival of Rabi oscillations in the JC model. The perfect entanglement reciprocation is a feature of a coherent state. We have considered a good cavity and detector limit. A longer memory will need a higher ratio between $\lambda$ and the cavity decay rate.

We thank the U.K. EPSRC, KRF (2003-070-C00024), the Leverhulme Trust (ECF/40157), and the Korean Ministry of Science and Technology through QPSRC.

[1] B. Reznik, quant-ph/0008006.

[2] W. Son, J. Lee, M. S. Kim, and D. Ahn, J. Mod. Opt. 49, 1739 (2002); M. Paternostro, W. Son, and M.S. Kim, Phys. Rev. Lett. 92, 197901 (2004); M. Paternostro et al., Phys. Rev. A 70, 022320 (2004).

[3] B. Kraus and J. I. Cirac, Phys. Rev. Lett. 92, 013602 (2004); A. Retzker, J. I. Cirac, and B. Reznik, Phys. Rev. Lett. 94, 050504 (2005).

[4] M.S. Kim, presented at 100 Years Werner Heisenberg: Works and Impact, Bamberg, 2002 (unpublished).

[5] S. J. van Enk, Phys. Rev. A 72, 064306 (2005).

[6] E. T. Jaynes and F.W. Cummings, Proc. IEEE 51, 89 (1963); B. W. Shore and P. L. Knight, J. Mod. Opt. 40, 1195 (1993).

[7] M. Paternostro, G. Falci, M. Kim, and G. M. Palma, Phys. Rev. B 69, 214502 (2004); I. Wilson-Rae and A. Imamoglu, Phys. Rev. B 65, 235311 (2002).

[8] S. J. D. Phoenix and P. L. Knight, Ann. Phys. (N.Y.) 186, 381 (1988).

[9] S. M. Barnett and P. M. Radmore, Methods in Theoretical Quantum Optics (Oxford, New York, 1997).

[10] We have also found the robustness of this scheme to small asymmetries of the Rabi frequencies. This is due to the fact that, for small differences, the functions generalizing $v_{0}$ to different $\lambda$ 's are simultaneously zero for a considerably long interaction time.

[11] J. Lee, M. S. Kim, Y. J. Park, and S. Lee, J. Mod. Opt. 47, 2151 (2000).

[12] The field injection is routinely performed in microwave cavities [13]. In the optical regime it is more convenient to mix the released field mode with a coherent state of a proper amplitude at a high-transmittivity beam splitter. This effectively realizes a field displacement.

[13] J. M. Raimond, M. Brune, and S. Haroche, Rev. Mod. Phys. 73, 565 (2001).

[14] S. Bose, I. Fuentes-Guridi, P. L. Knight, and V. Vedral, Phys. Rev. Lett. 87, 050401 (2001).

[15] G. S. Solomon, M. Pelton, and Y. Yamamoto, Phys. Rev. Lett. 86, 3903 (2001); A. Wallraff et al., Nature (London) 431, 162 (2004). 